\title{
Laryngeal Mucositis, CTCAE
}

National Cancer Institute

\section{Source}

National Cancer Institute. Laryngeal Mucositis, CTCAE. NCI Thesaurus. Code C143638.

A disorder characterized by ulceration or inflammation involving the mucous membrane

of the larynx. 